\title{
Epidemiología de la insuficiencia cardiaca. Proporciones de epidemia
}

\author{
R. DE LA FUENTE CID, A. HERMIDAAMEIJEIRAS, M. PAZO NÚÑEZ, F. LADO LADO
}

Servicio de Medicina Interna. Complejo Hospitalario Universitario Santiago de Compostela

\author{
EPIDEMIOLOGY OF HEART FAILURE. RATIO OF EPIDEMIC
}

\begin{abstract}
RESUMEN
La Insuficiencia Cardíaca (IC) es un síndrome complejo, con una alta prevalencia en la población, situándose en torno al $10 \%$ en mayores de 70 años, y esta continúa aumentando. Su incidencia en mayores de 65 años es del $1 \%$ al año. En países desarrollados es la primera causa de hospitalización en mayores de 65 años, suponiendo el 5\% del total de ingresos, y su porcentaje se incrementa cada año. La mayoría de los autores reconoce que las cifras alcanzan valores de epidemia, si bien es cierto que debido a su complejidad no es fácil de definir ni de cuantificar.

Aunque la supervivencia ha mejorado en los últimos años, continúa teniendo un mal pronóstico con una mortalidad aproximada del $50 \%$ a los 5 años del diagnóstico. Debido a su elevada prevalencia y a su alta tasa de ingresos-reingresos supone un problema de salud pública por su elevada carga asistencial. En conjunto se estima que los costes directos de la IC suponen 1-2\% del presupuesto sanitario de los países desarrollados.
\end{abstract}

PALABRAS CLAVE: Insuficiencia cardíaca. Epidemiología.

\begin{abstract}
The Heart Failure is a complex sindrom with a high prevalence in the population, being about $10 \%$ in older than 70 years and it is continuosly increasing. Its incidence in persons older than 65 years is about $1 \%$ per year. In developed countries it is the first cause of hospitalitation in older than 65 years, supposing the 5\% of total admissions and it is increasing every year. The most of authors recognise that the numbers reach values of epidemic, although it is true that it is difficult to determine and cuantify due to its complexity.

Although the supervivence has improved in last years, it continues being in a bad pronostic with a mortality about $50 \%$ after 5 years approximately of the diagnostic. In order to its high prevalence and its high rate of admissions-readmissions it supposes a problem of public health due to high assistance work. Globally it is estimated that the direct costs of heart failure suppose 1-2 \% of healthy cost of developed countries.
\end{abstract}

KEY WORDS: Heart failure. Epidemiology.

de la Fuente Cid R, Hermida Ameijeiras A, Pazo Núñez M, Lado Lado F. Epidemiología de la insuficiencia cardiaca. An Med Interna (Madrid) 2007; 24: 500-504.

\section{INTRODUCCIÓN}

Indudablemente cuando quieres abordar las dimensiones de una enfermedad, lo primero que debes de considerar son los criterios que la definen. La epidemiología está claramente influenciada por la existencia o no, de unos ajustados criterios diagnósticos (1). Así, por ejemplo en el estudio Framingham, (2) se utiliza un diagnóstico clínico (signos y síntomas) que es relativamente inespecífico. Si utilizamos la ecocardiografía nos permite conocer si existe disfunción ventricular sistólica, diastólica o si cursa de forma asintomática (3). Recientemente la FDA ha incluido un nuevo parámetro analítico, el péptido natriurético tipo $\mathrm{B}(\mathrm{BNP})$, en el diagnóstico de la insuficiencia cardíaca (IC), especialmente en pacientes no tratados (4). Es más que probable que ello haga variar las cifras de prevalencia e incidencia de la IC. Esta falta de unos criterios diagnósticos ajustados, provoca una gran variabilidad entre los distin- tos profesionales y/o centros; a ello deberíamos de añadir un desinterés científico hasta hace pocos años, por falta de tratamientos eficaces. Finalmente recordar la dificultad que entraña el asignar en el certificado de defunción como responsable última de la muerte, a la IC, al tratarse de una entidad que constituye el estadío final de muchas enfermedades a las que en ocasiones se les concede más importancia. Todo ello ha originado que exista un importante desconocimiento, hasta hace poco, de la epidemiología de esta enfermedad.

Además hemos de considerar la población que estamos estudiando y su ámbito geográfico. A este respecto conviene señalar que la mayoría de los estudios epidemiológicos están realizados en países anglosajones, y en pacientes con deterioro de la función sistólica (5). Esta dificultad en el método, cuando hablamos de insuficiencia cardíaca (IC) va a complicar el estudio comparativo, hecho que no debemos olvidar.

Trabajo aceptado: 10 de mayo de 2007

Correspondencia: Ramón de la Fuente Cid. Servicio de Medicina Interna. Complejo Hospitalario Universitario Santiago de Compostela. C/ San Ignacio, 1, 4 C. 15001 La Coruña. e-mail: ramondfc@yahoo.es 
Debido por un lado a los nuevos conocimientos en la fisiopatología que han originado avances terapéuticos que han permitido reducir las tasas de morbimortalidad (aunque persisten muy altas) y por otro lado al envejecimiento de la población, la IC se ha convertido en uno de los principales problemas de salud pública a nivel mundial (2).

\section{PREVALENCIA}

La prevalencia nos indica el número de casos que existen de una enfermedad en un período y en una población determinada. Sería muy costoso y dificultoso el poder realizar los estudios sobre la población general, por lo que habitualmente se llevan a cabo sobre subpoblaciones (generalmente de centros sanitarios) y luego se extrapolan los resultados al conjunto de la sociedad (1).

En el año 2003 la American Heart Association (AHA), publicó que se producían 4,8 millones de altas hospitalarias en Estados Unidos con este diagnóstico, aunque dependía de los criterios diagnóstico utilizados.

En el estudio Framingham, en el que el diagnóstico se realizó por parámetros clínicos, se observó que la IC en varones de 50 a 59 años era de 8/1.000 habitantes y que incrementaba hasta 70/1.000 en pacientes mayores de 80 años (cifras muy similares en mujeres) (2).

A este respecto debemos señalar, que existen estudios poblacionales en los que cerca del $50 \%$ de la población presentaba una fracción de eyección del ventrículo izquierdo (FEVI) inferior al $40 \%$ y no se encontraba diagnosticada de IC (6). En el otro lado de la moneda se encuentra el bajo valor predictivo de la sospecha clínica en atención primaria, al observarse que solo el $30 \%$ de los pacientes que se remitía para realización de ecocardiograma con dicho diagnóstico clínico, presentaba disfunción ventricular (7).

Con independencia del criterio que utilicemos para el diagnóstico, tanto la IC como la disfunción ventricular izquierda (DVI), aumenta progresivamente con la edad (8), y de forma general se asume que la prevalencia de la IC oscila entre el 1 y el 4\%(6).

Según la Sociedad Europea de Cardiología, con una población aproximada de 900 millones de personas, unos 10 millones de Europeos estarían afectadas, lo que en términos relativos significaría una prevalencia entre el 0,4 y 2\% (9).

En España, no existen muchos datos, pero en un estudio poblacional realizado en Asturias por Cortina et al y publicado en 2001, se observó una prevalencia media del $5 \%$, aunque esta era muy variable en función de la edad, pasando de ser inferior al $1 \%$ en menores de 50 años, en torno al $10 \%$ en sujetos mayores de 70 años y del $18 \%$ en mayores de 80 años (10). Aunque todavía no están publicados los resultados de un estudio realizado por el Dr. M. Anguita sobre prevalencia de IC en España, en la Revista Española de Cardiología en su suplemento $\mathrm{F}$ del volumen 6 , se reflejan algunos datos en los que se vuelve a confirmar un aumento de prevalencia con la edad, al pasar de $1,7 \%$ en el grupo de $45-54$ años a $6,1 \%$ en el grupo de 65-74 años y 18,7\% en el grupo de mayores de 75 años.

Este aumento de la prevalencia también se ha visto en el tiempo, pasando en personas de 75 años del $3 \%$ en el periodo 1976-80, a cerca del 10\% entre 1988-91 (11).

Varios factores han influido en ello, pero consideramos fundamental el envejecimiento progresivo de la población. En el mundo occidental han hecho su aparición las enfermedades crónicas, que como su nombre indica necesitan de muchos años para desarrollarse, naciendo la era de las enfermedades degenerativas demoradas, con patologías propias de personas de edades avanzadas. Debido a la mejoría en los estilos de vida de nuestra sociedad, la esperanza de vida ha aumentado de forma significativa en los últimos años. Según datos del Instituto Nacional de Estadística (INE), en España en el período de 1975-1998 la esperanza de vida al nacimiento, aumentó 5 años, pasando a ser de 75 años para el varón y 82 años para la mujer. En el año 2003, el 19,4\% de las mujeres y el 14,6\% de los varones tenía más de 65 años.

Los otros dos factores que han contribuido a este aumento de prevalencia son la mejora en el tratamiento de las dos entidades etiológicas fundamentales, como son la hipertensión arterial (HTA) y la cardiopatía isquémica, y finalmente la existencia de mejores tratamientos de la IC entre los que destacamos los IECA y betabloqueantes, lo que ha permitido que estos pacientes vivan más años y desarrollen IC en estadios más avanzados (12).

\section{INCIDENCIA}

La incidencia mide el número de casos nuevos de una enfermedad durante un determinado tiempo. Indirectamente nos está informando de la actividad que tienen los factores de riesgo causales de una patología sobre la población. Para ello debemos realizar estudios de seguimiento durante un tiempo determinado, sobre una población no afectada por la enfermedad y contabilizar los nuevos casos aparecidos.

También en esta ocasión, el estudio Framingham constituye el referente más importante y nos proporciona datos de gran valor (Tabla I). En él se observa que la incidencia aumenta progresivamente con la edad, llegando al $1 \%$ anual en personas mayores de 65 años. A partir de los 40 años, la incidencia se dobla por cada década que vivimos, con valores anuales para varones de 2 por mil en el grupo de 35 a 64 años, y de 12 por mil en el grupo de 65 a 94 años. Si nos referimos exclusivamente al grupo de 80-89 años, muestra una incidencia anual de 27 y 22 por mil para varones y mujeres respectivamente. Así pues parece claro que tanto la prevalencia como la incidencia aumenta con la edad (8).

En lo referente al riesgo de por vida de presentar IC, o planteado de otra forma, ¿cúal es el riesgo que tiene una persona de desarrollar IC en lo que le queda de vida? A los 40 años, las probabilidades para el varón son del $21 \%$ y para la mujer $20 \%$ (13).

\section{TABLA I}

INCIDENCIA ANUAL POR 1000

\begin{tabular}{lcc}
\hline Edad & $\begin{array}{c}\text { Cohorte de la población del estudio Framingham } \\
\text { Varones }\end{array}$ & Mujeres \\
\hline $45-54$ & 2 & 1 \\
$55-64$ & 4 & 2 \\
$65-74$ & 9 & 6 \\
$75-84$ & 18 & 12 \\
$85-94$ & 39 & 31 \\
\hline
\end{tabular}

Fuente: Eur. Heart J. 
Además de la edad, si analizamos los otros dos factores etiológicos que influyen de forma importante en su origen, podemos apreciar que el hecho de ser hipertenso multiplica por dos la incidencia, y el haber sufrido un IAM por cinco (2).

En EE.UU. se calcula que unas 400.000 personas son diagnosticadas anualmente de insuficiencia cardíaca (14).

En Europa, y también utilizando los criterios de Framingham, en un estudio realizado en Finlandia se objetivó una incidencia anual de 4,1 por 1.000 en varones y 1,6 por mil en mujeres (15).

No disponemos de datos poblacionales sobre la incidencia de la IC en España.

Cuando se analiza por sexos, en un estudio de Levy D et al, se observa que en ambos sexos aumenta con la edad, siendo 1,8 veces superior en varones (16).

Aunque la prevalencia, por lo anteriormente comentado, está aumentando, no parece estar tan clara esta tendencia con respecto a la incidencia. En la cohorte original del estudio Framingham, con 10.311 pacientes y cuando se realiza un ajuste por edad, se objetivó que la incidencia se encuentra estabilizada para varones desde los años 50 hasta la actualidad, y disminuyó en mujeres en el período 19501999 (16).

Otro estudio realizado en EE.UU., sobre la población censada en el condado de Olmsted, en Rochester, entre los años 1970-2000, no apreció cambios de incidencia respecto al sexo, ni tampoco diferencias significativas de aumento o disminución a lo largo del período de seguimiento (17).

A la vista de los resultados de los últimos años, en los que parece haber una incidencia mantenida, hemos de pensar que existe un mejor control de la HTA y un mejor manejo de la cardiopatía isquémica, lo que permitiría que menos enfermos desemboquen en el estadío de IC sintomática, aunque en el otro lado de la moneda nos encontramos con que aumenta la supervivencia de la población y en consecuencia exista un mayor número de personas expuestas a desarrollar ICC (18).

\section{MORTALIDAD-SUPERVIVENCIA}

La IC es un proceso progresivo que conduce a la muerte a pesar de un adecuado tratamiento.

Para poder realizar estudios sobre mortalidad/supervivencia en IC es preciso hacer un seguimiento de aquellos sujetos afectados por la enfermedad y que pertenecen a la población general, también podemos obtener información de registros hospitalarios y en otros casos mediante intervenciones en ensayos clínicos. No debemos de olvidar que los resultados obtenidos en estos últimos, son generalmente mejores puesto que los pacientes seleccionados suelen ser más jóvenes, cumplen unos criterio de inclusión específicos y tienen un seguimiento más estrecho.

En los estudios poblacionales, los datos de mortalidad se obtienen a partir de los certificados de defunción. Las normas de codificación, no están bien establecidas ya que puede ser la causa de la muerte una IC de origen isquémico y sin embargo se prioriza esta última coma causa fundamental del fallecimiento.

En EE.UU., con el envejecimiento de la población, las tasas de mortalidad por IC ajustadas por edad han aumentado de 3,8 por 100.000 en el año 1970 hasta el 7,7 por 100.000 en 1988, para ambos sexos (19).

Cuando las cifras se expresan con respecto a pacientes diagnosticados de IC, igualmente se objetiva un incremento de mortalidad de 5,8 por mil en 1970 a 16,4 por mil en 1993 (20).

La mortalidad no es uniforme durante todo el año, y tiene un claro patrón estacional con un aumento neto en los meses de invierno. La variabilidad es tan importante que puede llegar al $35 \%$ cuando se comparan los meses de enero y agosto (21).

Así pues y de un modo muy general podemos decir que la ICC tiene un pronóstico malo, ya que la supervivencia al año de diagnóstico es entre un 55-70\% y como se demostró en el estudio Framingham del 50\% a los 5 años (22).

Indudablemente estas cifras están sometidas a una gran variabilidad, dependiendo fundamentalmente de la edad y la comorbilidad. En un estudio realizado en Canadá con unos 38.000 pacientes con seguimiento durante cuatro años se observó, que en pacientes de 25 a 49 años y sin comorbilidad presentaban una mortalidad al año del diagnóstico del 12,9\% frente al $60,7 \%$ que presentaban los mayores de 75 años y más de tres enfermedades acompañantes (23). También en la población del estudio Framingham, se puso de manifiesto la relación existente entre aumento de edad y mortalidad de pacientes diagnosticados y tratados por ICC (24).

Otro factor que influye en el pronóstico es el pertenecer a uno u otro sexo. Una vez más en el Framingham se observa una supervivencia media tras el diagnóstico de 3,2 años para mujeres y 1,7 para los varones (24). La supervivencia a los cinco años fue del $60 \%$ en mujeres frente al $46 \%$ en varones (16).

Se analizó también la etiología como factor influyente en el pronóstico. En un estudio publicado en el 2000 por Felker et al., en el que se estudió a 1230 pacientes con un seguimiento medio de 4,4 años y en el que la miocardiopatía dilatada le sirvió de grupo control, observó que la causa que presentaba un peor pronóstico era la hemocromatósica y la amiloidótica, seguidas en orden decreciente por la miocardiopatia producida por VIH, posquimioterapia (doxurrubicina), enfermedad coronaria, conectivopatías y periparto. Los casos de HTA, miocarditis y sarcoidosis presentaban una mortalidad similar al grupo control (25).

Sin embargo en un estudio poblacional publicado por Levy et al en 2002, se observa una disminución de la mortalidad en los últimos años y que afecta tanto a varones como a mujeres (16). Resultados similares se aprecian en el análisis del estudio Framingham, donde aparece una mejoría en la supervivencia desde finales de los ochenta y de una manera más marcada desde 1990. Comparando los períodos comprendidos entre los años 1950-1969 y 1990-1999 se observó que la mortalidad al año, se redujo del 30 al $28 \%$ para varones y del 28 al $24 \%$ en mujeres. A los cinco años, la mortalidad se redujo del 70 al $59 \%$ en varones y del 57 al $45 \%$ en mujeres(16).

En Europa, en un estudio realizado en Escocia sobre mortalidad hospitalaria, se confirman estos datos con una discreta tendencia a aumentar la supervivencia a partir de principios de los noventa (26).

En España, según datos del año 2000, la IC fue la causante del $4 \%$ de defunciones en varones y el $8 \%$ en mujeres. $\mathrm{Al}$ igual que en el resto del mundo occidental, parece confirmarse en las últimas décadas una tendencia progresiva de la mor- 


\begin{tabular}{|c|c|c|c|c|c|c|c|c|c|}
\hline \multicolumn{10}{|c|}{ TABLA II } \\
\hline \multicolumn{10}{|c|}{$\begin{array}{c}\text { TASAS DE MORTALIDAD POR } 100.000 \text { HABITANTES. } \\
\text { ESPAÑA, AÑO } 2003\end{array}$} \\
\hline Edad & $0-9$ & $10-19$ & $20-29$ & $30-39$ & $40-49$ & $50-59$ & $60-69$ & $70-79$ & $>80$ \\
\hline Varones & 0.15 & 0.08 & 0.5 & 1.7 & 4.4 & 8.5 & 23.5 & 101.5 & 721.8 \\
\hline Mujeres & 0.05 & 0.22 & 0.24 & 0.43 & 1.3 & 3.5 & 13.7 & 84.8 & 958.3 \\
\hline
\end{tabular}

Fuente: Revista Española de Cardiología.

talidad hacia la disminución, aunque en el caso de las mujeres no parece tan claro (18). En personas mayores de 85 años dicha tendencia se encuentra estabilizada. Aunque las tasas de mortalidad son cada vez menores, el número total de fallecimientos por IC en mujeres ha aumentado, debido al envejecimiento de la población y al incremento del tamaño poblacional (27) (Tabla II).

Otro dato positivo y que nos invita al optimismo, lo encontramos en un estudio realizado por Polanczyc et al. en Massachussets durante los años 1986-1996, en el que se examinó la mortalidad hospitalaria por ICC y en el que se encontró una disminución de esta del 8,4 al 6\% (28).

Es más que probable que esta mejoría en la supervivencia viene determinada en gran medida por los nuevos fármacos aparecidos en el tratamiento de la IC y su mayor utilización. Los ensayos clínicos que comparan inhibidores de la enzima de conversión de la angiotensina (IECA) frente a placebo demuestra una mejora de la supervivencia del 20 y del $35 \%$ en el caso de los betabloqueantes, independientemente de la clase funcional $(29,30)$.

Los pacientes tratados por cardiólogos reciben más IECA, 83 frente a $68 \%$, y betabloqueantes 38 frente a $22 \%$, que los tratados por médicos generales. Ello se traduce, tras el análisis multivariante, en que los pacientes tratados por cardiólogos tienen menor riesgo de muerte u hospitalización (31).

En el caso de pacientes ambulatorios tratados mediante programas de manejo multidisciplinario, no han demostrado mejoría en la supervivencia (32).

Así pues parece clara la tendencia a mejorar la supervivencia en los últimos años, no obstante no debemos de olvidar que en los pacientes con IC por disfunción diastólica o alteraciones valvulares está por demostrar la eficacia de IECA y betabloqueantes. En los casos que presentan FE deprimida, los niveles de fármacos que aumentan la supervivencia, presentan unos índices bajos de prescripción. Finalmente recordar de nuevo que muchos de los resultados obtenidos en ensayos clínicos sobre mortalidad, están maquillados al excluir de ellos en muchas ocasiones a pacientes pluripatológicos, añosos e incluso mujeres (33).

\section{HOSPITALIZACIONES}

Junto con la prevalencia, las hospitalizaciones son un índice interesante de la carga asistencial que supone este problema de salud. La información se obtiene de los sistemas de codificación sanitarios, lo que constituye una manera cómoda y sencilla de obtener los resultados. Los ingresos (que son los responsables de la mayor parte del gasto sanitario que genera la IC) van a depender de los recursos sanitarios disponibles en esa determinada zona y en consecuencia muy variables de unos lugares a otros (18).

De una forma muy general, podríamos decir que de todos los pacientes diagnosticados de IC, el $35 \%$ son hospitalizados por este problema cada año (34).

Al analizar las cifras de hospitalizaciones, debemos de tener en cuenta determinantes tan importantes como la nueva concepción de nuestra sociedad sobre la enfermedad terminal y la utilización de los recursos sanitarios, la probable tendencia al incremento de diagnósticos finales en los informes de alta con el fin de incrementar la complejidad de estos, el aumento en la esperanza de vida y por tanto de personas mayores de 65 años, el aumento de camas hospitalarias en los últimos años, o incluso como en el caso de EE.UU. el hecho de que la mayoría de los seguros médicos son privados.

En EE.UU. la tasa de hospitalizaciones por IC ha aumentado en más del triple desde 1970 hasta 1995, y de forma más importante en el grupo de personas mayores de 65 años y con comorbilidad asociada, siendo esta última la causa más importante que condiciona el ingreso (35).

En el año 1994 y sobre un total de 874.000 altas hospitalarias, la IC fue el primer diagnóstico. El 20\% de los ingresos hospitalarios en personas mayores de 65 años es debido a IC o participa en gran medida en dicho diagnóstico (36).

En Europa Occidental, las cifras son similares; en un estudio publicado en 1996 y realizado durante los años 1980-1993, se objetivó un incremento de las tasas de hospitalización por IC del $48 \%$ para varones y del $40 \%$ para mujeres (37).

En España, al igual que en Europa Occidental, la IC es la primera causa de hospitalización en personas mayores de 65 años, que corresponde a unos 80.000 ingresos anuales, lo que supone un $5 \%$ del total y con un marcado componente estacional, en el que durante los meses de invierno existe un aumento manifiesto (38).

En España como en el resto de países desarrollados los ingresos por IC han aumentado en los últimos años, y es más que probable que lo siga haciendo debido al envejecimiento poblacional. Dicho incremento de hospitalizaciones es más marcado entre las mujeres, superando en la actualidad a los originados por los varones (39).

\section{CONCLUSIONES}

La mayoría de los autores consideran que la IC presenta dimensiones de epidemia, y lejos de ser controlada esta sigue su expansión. Ello es debido a que por un lado el número de personas mayores de 65 años es cada vez mayor en países desarrollados y por otro lado a que la supervivencia por IC ha mejorado en los últimos años. En consecuencia la prevalencia y la tasa de hospitalizaciones, considerados los dos parámetros que mejor reflejan la carga asistencial, continúan aumentando. La manera más razonable de poder controlar el problema sería conseguir un descenso en la incidencia, con lo cual deberíamos de actuar sobre los factores de riesgo potenciales de desarrollar IC, que continúan siendo la hipertensión arterial y la cardiopatía isquémica. 


\section{Bibliografía}

1. Cowie MR. Annotated references in epidemiology. Eur J Heart Fail 1999; 1: 101-7

2. Ho KK, Pinsky JL, Kannel WB, Levy D. The epidemiology of heart failure: the Framingham Study. J Am Coll Cardiol 1993; 22: 6A-13A.

3. Vasan RS, Levy D. Defining diastolic Herat failure. A call for standardized diagnostic criteria. Circulation 2000; 101; 2118-21.

4. Remme WJ, Swedberg K. Task Force for the Diagnosis and Treatment of Chronic Heart Failure, European Society of Cardiology. Guidelines for the diagnosis and treatment of chronic heart failure. Eur Heart $\mathrm{J}$ 2001; 22: 1527-60.

5. Konstam MA. Systolic and diastolic disfunction in heart failure. Time for a new paradigm. J Card Fail 2003; 9: 1-3.

6. Redfield MM, jacobsen SJ, Burnett JC Jr, Mahoney DW, Bailey KR, Rodeheffer RJ. Burden of systolic and diastolic ventricular dysfunction in the community: Apreciating the scope of the heart failure epidemic. JAMA 2003; 289: 194-202.

7. Cowie MR, Struthers AD, Wood DA, Coats AJ, thompson SG, PooleWilson PA, et al. Value of natriuretic peptides in assessment of patients with possible new heart failure in primary care. Lancet 1997; 350: 1349-53.

8. Bleumink GS, Knetsch AM, Sturkenboom MC, Straus SM, Hofman A, Deckers JW, et al. Quantifying the heart failure epidemic: Prevalence, incidence rate, lifetime risk and prognosis of heart failure The Rotterdam Study. Eur Heart J 2004; 25: 1614-9.

9. Hunt SA, Beker DW, Chin MH, Cinquegrani MP, Feldman AM, Francis GS et al. ACC/AHA guidelines for the evaluation and management of chronic heart failure in the adult: Executive sumary. A report of the Americam College of Cardiology/ Americam Heart Association Task Force on Practice Guidelines. J Am Coll Cardiol 2001; 38: 2001-13.

10. Cortina A, Reguero J, Segovia E, Rodríguez Lambert JL, Cortina R, Arias JC et al. Prevalence of Herat failure in Asturias. Am J Cardiol 2001; 87: 1417-19.

11. McKee PA, Castelli WP, McNamara PM, Kannel WB, The natural history of congestive heart failure: The Framingham study. N Engl J Med 1971; 285: 1441-46

12. Cleland JGF, Gemmel I, Khand A, Boddy A. Is the prognosis of heart failure improving? Eur J Heart Fail 1999; 1: 229-41.

13. Lloyd-Jones DM, Larson MG, Leip EP, Beiser A, D'Agostino RB, Kannel WB, et al. Lifetime rick for developing congestive Heart Failure: the Framingham Heart Study: Circulation 2002; 106: 3068-72.

14. Dargie HJ, McMurray JJ, McDonagh TA. Heart failure implications of the true size of the problem. J Intern Med 1996; 239: 309-15.

15. Eriksson H, Svardsudd K, Larsson B, Ohlson LO, Tibblin G, Welin L et al. Risk factors for heart failure in the general population: the study of men born in 1913. Eur Heart J 1989; 10: 647-56.

16. Levy D, Kenchaiah S, Larson MG, Benjamin EJ, Kupka MJ, Ho KK et al. Long-term trends in the incidence of and survival with heart failure. N Engl J Med 2002; 347: 1397-402.

17. Roger VL, Weston SA, Redfield MM, Hellermann-Homan JP, Killian J, Yawn BP, et al. Trends in heart failure incidence and survival in a community-based population JAMA 2004; 292: 344-50.

18. Rodríguez-Artalejo F, Banegas Banegas JR, Guallar-Castillón P. Epidemiología de la Insuficiencia Cardíaca. Rev Esp Cardiol 2004; 57: 16370 .

19. Adams KF, Jr. New epidemiologic perspectives concerning mild-tomoderate heart failure. Am J Med 2001; 110 (Supl. 7A): 6S-13S .

20. National Heart Lung and Blood Institute. Morbidity and Mortality
Chart-book on cardiovascular, Lung and blood Disease. Bethesda: National Institutes of Health; 1996.

21. Boulay F, Berthier F, Sisteron O, Gendreike Y, Gibelin P. Seasonal variation in chronic heart failure hospitalizations and mortality in France. Circulation 1999; 100: 280-6.

22. Mosterd A, Cost B, Hoes AW, de Bruijne MC, Deckers JW, Hofman A, et al. The prognosis of heart failure in the general population. The Rotterdam study. Eur Heart J 2001; 22: 1318-27.

23. Jong P, Vowinckel SJ, Liu PP, Gong Y, Tu JV. Prognosis and determinants of suvirval in patients newly hospitalizad fpr Herat failure: a population-based study. Arch Intern Med 2002; 162: 1689-94.

24. Ho KK, Anderson KM, Kannel WB, Grossman W, Levy D. Survival after the onset of congestive heart failure in Framingham Heart Study subjects. Circulation 1993; 88: 107-15.

25. Felker CM, Thompson RE, Hare JM, Hruban RH, Clemetson DE, Howard DL, et al. Underlying causes and long-term survival in patients with initially unexplained cardiomyopathy. N Eng J Med 2000; 343: 1077-84.

26. MacIntyre k, capewell S, Stewart S, Chalmers JW, Boyd J, Finlayson A, et al. Evidence of improving prognosis in heart failure: Trends in case fatality in 66,547 patients hospitalized between 1986 and 1995. Circulation 2000; 102: 1076-8.

27. Boix Martínez R, Almazán Isla J, Medrano Albero MJ. Mortalidad por insuficiencia cardíaca en España 1977-1998. Rev Esp Cardiol 2002; 55: 219-26.

28. Polanczyc CA, Rhode LE, Dec GW, diSalvo T. ten-year trenes for 23,505 Medicare patients hospitalizad with Herat failure in northeast Ohio, 1991 to 1997: Am Heart J 2003; 146: 258-64.

29. SOLVD Investigators. Effect of enalapril on mortality and the development of heart failure in asymptomatic patients with reduced left ventricular ejection fraction. N Engl J Med 1992: 327: 685-91.

30. Packer M, Coats AJ, Fowler MB, Katus HA, Krum H, Mohacsi P, et al. Effect of carvedilol on survival in severe chronic heart failure. N Engl J Med 2001; 344: 1651-8.

31. Ansari M, Alexander A, Tutar A, Bello D, Massie BM. Cardiology participation improves outcomes in patients with new-onset Herat failure in the outpatients setting. J Am Coll Cardiol 2003; 41: 62-8.

32. McAlister FA, Lawson FM, Teo KK, Armstrong PW. A systematic review of randomized trials of disease management programs in heart failure. Am J Med 2001; 110: 378-84.

33. Martínez-Sellés M, García Robles JA, Prieto L, Frades E, Muñoz R, Díaz Castro $\mathrm{O}$, et al. Características de los pacientes ingresados por insuficiencia cardíaca según el estado de su función ventricular. Rev Esp Cardiol 2002; 55: 579-86.

34. Abel-Smith B. Global perspective on health service financing. Soc Sci Med 1985; 21: 957-63.

35. Brown AM, Cleland JG. Influence of concomitant disease on patterns of hospitalitation with heart failure discharged from Scottish hospitls in1995. Eur Heart J 1998; 19: 1063-9.

36. Jessup M, Brozena S. Heart Failure. N Engl J Med 2003; 384: 2007-18.

37. Reitsma JB, Mosterd A, de Craen AJ, Koster RW, van Capelle FJ, Grobbee DE, et al. Increase in hospital adamission rates for heart failure in Netherlands, 1980-1993. Heart 1996; 76: 388-92.

38. Martínez-Selles M, García Robles JA, Prieto L, Serrano JA, Muñoz R, Frades E, et al. Annual Rates of admisión and seasonal variations in hospitalizations for heart failure . Eur J Heart Fail 2002; 4: 779-86.

39. McMurray JJ, Stewart S. Heart Failure. Epidemiology, aetiology, and prognosis of heart failure. Heart 2000; 83: 596-602. 